\title{
Aortic reinterventions after the frozen elephant trunk procedure
}

Maximilian Kreibich, MD, Tim Berger, MD, Bartosz Rylski, MD, Zehang Chen, BA, Friedhelm Beyersdorf, MD, Matthias Siepe, MD, and Martin Czerny, MD, MBA

\section{ABSTRACT}

Objective: The frozen elephant trunk (FET) procedure has emerged as a potential single-step treatment for pathologies of the thoracic aorta, but the procedure's true potential to be a single-step treatment remains unclear. The aim of this study was to evaluate the need and outcomes of aortic reinterventions after previous FET implantation.

Methods: Patient characteristics and follow-up data of 107 patients following the FET procedure were evaluated and compared between patients with and without aortic reinterventions. A competing risk regression model was analyzed to identify independent predictors of aortic reintervention and to predict the risk for reintervention.

Results: Intended completion, anticipated reinterventions, and unexpected reinterventions were performed in 35 patients $(33 \%)$. There was no difference in the underlying pathology between patients with or without aortic reintervention. An endovascular reintervention was performed in 24 patients $(69 \%)$, open surgery in 7 patients $(20 \%)$ and a hybrid approach in 4 patients $(11 \%)$. No stroke or permanent spinal cord injuries were observed. In-hospital mortality after reintervention was $14 \%$ (5 patients), but there was no difference in survival during follow-up after FET implantation ( $\log$ rank test, $P=.58$ ). No risk factors for aortic reinterventions were identified. The risk for aortic reintervention was $31 \%(95 \%$ confidence interval [CI], 21\%-42\%), 49\% (95\% CI, 35\%-62\%), and 64\% (95\% CI, $44 \%-79 \%$ ) after 12,24 , and 36 months, respectively.

Conclusions: Aortic reinterventions are common and likely after FET implantation, but this study did not identify independent predictors. Reinterventions are associated with acceptable morbidity and mortality. Close follow-up of all patients undergoing FET procedure is paramount. ( $\mathrm{J}$ Thorac Cardiovasc Surg 2020;159:392-9)

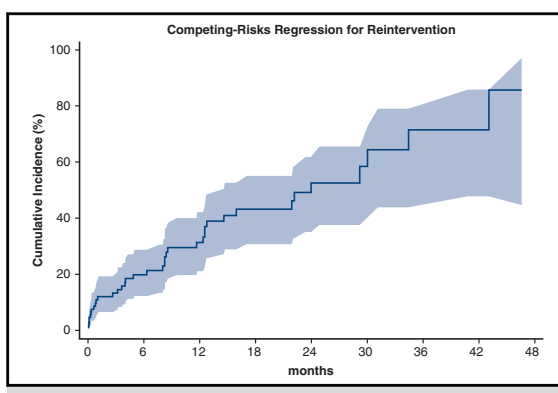

Aortic reinterventions after the frozen elephant trunk procedure are very likely.

\section{Central Message}

Close follow-up of patients after frozen elephant trunk procedure independent of the underlying disease is paramount because of the high risk for aortic reinterventions.

\section{Perspective}

Reinterventions after the frozen elephant trunk (FET) procedure are likely and common. Although morbidity and mortality of the reinterventions are relatively low, the causes are manifold and the device does not seem to be ideally designed for all aortic pathologies. Because of the high risk for aortic reinterventions, close follow-up of all patients undergoing FET independent of the underlying disease is paramount.

See Commentary on page 400 .
The frozen elephant trunk (FET) procedure is an effective surgical treatment option for different proximal thoracic aortic pathologies involving the distal arch and the descending aorta. ${ }^{1-3}$ It provides a proximal platform for second-

From the Department of Cardiovascular Surgery, University Heart Center Freiburg, Freiburg, Germany; and Faculty of Medicine, University of Freiburg, Freiburg, Germany.

Supported by institutional funding.

Received for publication Sept 4, 2018; revisions received Feb 4, 2019; accepted for publication Feb 17, 2019; available ahead of print March 28, 2019.

Address for reprints: Maximilian Kreibich, MD, University Heart Center Freiburg, Hugstetter Str 55, 79106 Freiburg, Germany (E-mail: maximilian.kreibich@ universitaets-herzzentrum.de).

$0022-5223 / \$ 36.00$

Copyright (c) 2019 by The American Association for Thoracic Surgery

https://doi.org/10.1016/j.jtcvs.2019.02.069 stage endovascular or surgical (ie, thoracoabdominal) repair and may potentially be used to treat the thoracic aorta in 1 single step. ${ }^{3,4}$ Although the FET technique facilitates positive remodeling in downstream aortic segments in a variety of different aortic pathologies, the need for aortic reinterventions following the FET procedure and the potential of the FET technique to truly be a single-step

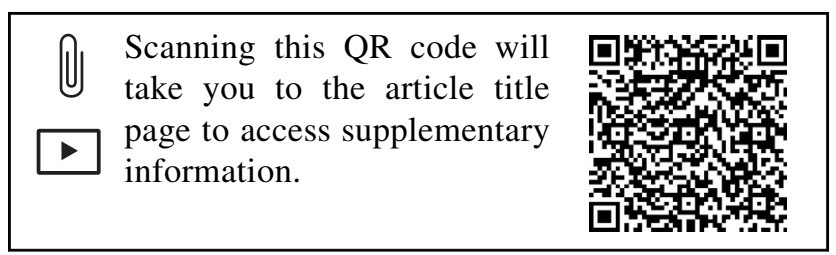




\section{Abbreviations and Acronyms}

CTA = computed tomography angiography

$\mathrm{dSINE}=$ distal stent-graft induced entry

FET $=$ frozen elephant trunk

TEVAR $=$ thoracic endovascular aortic repair

procedure to treat the thoracic aorta remain unclear. ${ }^{5-7}$ Therefore, the aim of this study was to evaluate the need and outcomes of aortic reinterventions after previous FET implantation.

\section{PATIENTS AND METHODS \\ Patients and Follow-up Protocol}

Between March 2013 and December 2017, 116 patients underwent the FET procedure in our center. Of those, 9 patients died during surgery (1 patient died because of descending aortic rupture) or within the immediate postoperative period ( 5 patients died because of major stroke, 1 died as a result of intracranial bleed, and 2 patients because of acute heart failure) before computed tomography angiography (CTA) scans of the aorta were performed. These 9 patients were excluded from this study. Therefore, the study population consists of 107 patients who underwent the FET procedure. Follow-up was available in all 107 patients after FET implantation $(100 \%)$. Patients were followed-up for a total of 118 patient-years, with a median follow-up of 0.7 years (first quartile, third quartile: $0.1,1.8$ years). All patients were routinely followed-up in our outpatient aortic clinic after 6 months, 12 months, and yearly thereafter. CTA scans were performed in all patients preoperatively, before discharge, during every clinic visit, and when clinically warranted. Aortic remodeling (including aortic diameters, false lumen thrombosis, distal perfusion, and endoleakage) was analyzed in every postoperative CTA as previously described. ${ }^{6}$ During this period, 39 aortic reinterventions were performed in 35 of those patients. In 31 of these patients, a single aortic reintervention was performed and in 4 patients a hybrid approach was performed with endovascular distal extension of the FET stent-graft shortly followed by open thoracoabdominal replacement ( 2 reinterventions per patient). Early outcomes were evaluated. The institutional review committee approved this retrospective study and the need for informed consent was waived.

\section{Outcome Measures}

Data were collected retrospectively using our center's prospectively maintained aortic database. Aortic dissection was classified as acute when the onset occurred fewer than 14 days before clinical presentation ${ }^{8}$ and were otherwise classified as chronic. Acute non-A non-B aortic dissection was defined as an aortic arch dissection with an entry distal to the left subclavian artery (descending-entry type) or an entry between the innominate and left subclavian arteries (arch-entry type) without involvement of the ascending aorta. ${ }^{9}$ Early diameter increase was defined as an aortic diameter increase requiring reintervention during the index hospitalization, and late diameter increase was defined as an aortic diameter increase requiring reintervention after the index hospitalization. All patients with significant diameter increase underwent aortic reintervention. A distal stent-graft induced entry (dSINE) was defined as a new tear caused by the stent-graft of the FET prosthesis, excluding tears created by natural disease progression or any iatrogenic injury. ${ }^{10}$ Graft infection was diagnosed clinically in conjunction with blood tests and imaging results. ${ }^{11}$

\section{Surgical Technique}

Our indications and standardized integrated surgical management for the FET technique have previously been described. ${ }^{1,2,4,6,9,12}$ In short, the axillary artery was cannulated in all patients and the entire aortic arch was replaced with the FET prosthesis. Lower body hypothermic circulatory arrest (target temperature, $25^{\circ} \mathrm{C}-26^{\circ} \mathrm{C}$ ) and selective antegrade cerebral perfusion were used in all patients. We routinely use the short $(100 \mathrm{~mm})$ version of the Thoraflex Hybrid graft (Vascutek Terumo, Renfrewshire, Scotland) in all patients and perform metachronous distal extension as second-stage completion if required. Intended early reinterventions were performed in 5 patients with extensive downstream aortic aneurysmatic dilatation $(\mathrm{n}=1)$, aortic dissection, and unsuccessful sealing of the primary entry tear or several communications between the 2 lumina in the thoracic aorta creating a high risk of early aortic growth $(n=4)$. Secondary reinterventions in the downstream aorta were preferably performed endovascularly if suitable. Open repair was performed in patients with connective tissue disorders, extensive kinking or atherosclerotic plaques within the abdominal aorta or iliac arteries, or patients without a suitable distal landing zone. In those cases, left heart bypass was used for replacement of the descending thoracic aorta or in cases of thoracoabdominal aortic replacement. In case of FET graft infection an integrated standardized therapeutic concept was applied, consisting of complete removal of the infected prosthetic material, extensive debridement, and orthotopic vascular reconstruction with self-made tube grafts constructed from bovine pericardial patches as previously described. ${ }^{11} \mathrm{~A}$ hybrid procedure was defined as endovascular distal extension of the FET stent-graft shortly followed by open thoracoabdominal replacement as a planned intervention to prevent spontaneous perioperative leakage of the FET stent-graft portion ${ }^{13}$ and to improve surgical exposure of the proximal anastomosis.

\section{Statistical Analysis}

Data are presented as absolute and relative frequency or as median (first quartile, third quartile). A student $t$ test or the Mann-Whitney $U$ test were used to compare continuous variables as appropriate. Categorical variables were compared using the $\chi^{2}$ test with the calculation of exact values. In case of small group sizes $(n<5)$, Fisher exact test was used. Survival was analyzed and compared using the Kaplan-Meier method and the log rank test. A competing risk analysis was performed to analyze the influence of selected variables (eg, age, sex, bicuspid aortic valve, Marfan syndrome, previous aortic intervention, and the underlying aortic pathology) on the risk for aortic reintervention. The statistical analysis was performed using IBM SPSS Statistics version 21.0 (IBM-SPSS Inc, Armonk, NY) and SAS version 9.4 (SAS Institute Inc, Cary, NC).

\section{RESULTS}

\section{Patient Characteristics}

Aortic reinterventions were performed in 35 patients $(33 \%)$. There was no statistically significant difference in patient characteristics between patients with or without aortic reinterventions except of a significantly higher incidence of chronic renal impairment in patients without aortic reinterventions $(P=.03)$. All patient demographic characteristics and risk factors are summarized in (Table E1).

\section{Aortic Characteristics}

Of 107 patients, 71 patients $(66 \%)$ were treated with the FET procedure because of acute or chronic aortic dissections and one quarter of all patients were treated because of a significant aortic aneurysm. Ten patients were treated for a penetrating atherosclerotic ulceration of the distal aortic arch. One patient was treated for recurring stenosis 
TABLE 1. Underlying pathology

\begin{tabular}{lcccr}
\hline \multicolumn{1}{c}{ Pathology } & $\begin{array}{c}\text { All } \\
(\mathbf{N}=\mathbf{1 0 7})\end{array}$ & $\begin{array}{c}\mathbf{R}+ \\
(\mathbf{n}=\mathbf{3 5})\end{array}$ & $\begin{array}{c}\mathbf{R}- \\
(\mathbf{n}=\mathbf{7 2})\end{array}$ & $\begin{array}{c}\boldsymbol{P} \\
\text { value }\end{array}$ \\
\hline Acute aortic dissection & $31(29)$ & $10(29)$ & $21(30)$ & .95 \\
Type A & $15(14)$ & $4(11)$ & $11(15)$ & 1.00 \\
Type non-A non-B & $6(6)$ & $2(6)$ & $4(6)$ & .97 \\
Type B & $10(9)$ & $4(11)$ & $6(8)$ & .61 \\
Chronic aortic dissection & $40(37)$ & $15(43)$ & $25(35)$ & .67 \\
Type A* & $30(28)$ & $12(34)$ & $18(25)$ & .32 \\
Type non-A non-B & $4(4)$ & $1(2)$ & $3(4)$ & .67 \\
Type B & $6(7)$ & $2(6)$ & $4(6)$ & .97 \\
Aortic aneurysm & $25(23)$ & $7(20)$ & $18(25)$ & .66 \\
PAU & $10(9)$ & $3(9)$ & $7(10)$ & 1.00 \\
Aortic coarctation & $1(1)$ & $0(0)$ & $1(1)$ & 1.00 \\
\hline
\end{tabular}

of the aortic arch following multiple unsuccessful previous interventions for congenital aortic coarctation. The underlying aortic pathologies are summarized in Table 1 . There was no statistical difference between patients with or without aortic reinterventions. In patients with aortic dissection, postoperative CTA scans following FET implantation revealed considerably lower rates of false lumen thrombosis in patients requiring aortic reinterventions: at the level of the stent-graft $(42 \%$ vs $70 \% ; P=.05)$, at the level of the thoracoabdominal transition $(11 \%$ vs $38 \% ; P=.037)$, and at the celiac trunk level $(11 \%$ vs $28 \% ; P=.189)$. However, there was no statistical difference in the distal extent of the aortic dissection between patients with or without aortic reintervention (Table E2).

\section{Indications for Aortic Reinterventions}

Table 2 provides an overview of all indications for aortic reinterventions. A second-stage completion was intended before FET implantation in 5 patients $(13 \%)$. Most patients (17 patients; 44\%) were operated on for aortic diameter progression. An endoleak was detected in one quarter of all patients (9 patients; $23 \%$ ) that required aortic reintervention. A dSINE was detected in 4 patients $(11 \%)$ requiring aortic reintervention and significant kinking of the stent-graft was observed in 2 patients $(5 \%)$. One patient (3\%) developed a FET stent-graft infection and another patient $(3 \%)$ developed thromboembolic lower body malperfusion requiring reintervention.

\section{Surgical Details}

Aortic reinterventions were performed after 12 months (2, 32 months) following the FET procedure. In 24 patients $(69 \%)$, an endovascular reintervention was performed. In 23 of those patients thoracic endovascular aortic repair (TEVAR) was the treatment and in 1 patient isolated salvage reintervention for thromboembolic occlusion of the mesenteric and renal arteries was performed. Open thoracic, thoracoabdominal aortic, or abdominal aortic replacements were performed in 7 patients $(20 \%)$ ). A conventional vascular graft was sewn to the stent-graft portion of the FET prosthesis (5 patients) or to the conventional polyester portion of the FET prosthesis (1 patient). One patient was treated with infrarenal aortic replacement because of a true infrarenal abdominal aortic aneurysm. The patient was initially treated for acute non-A non-B aortic dissection with the FET technique. The dissection ended just above the true abdominal aortic aneurysm and there was complete

TABLE 2. Indications for aortic reinterventions*

\begin{tabular}{|c|c|c|c|c|c|c|}
\hline \multirow[b]{2}{*}{ Indication } & \multirow[b]{2}{*}{$\mathbf{R}+(\mathbf{n}=\mathbf{3 5})$} & \multicolumn{4}{|c|}{ Underlying pathology } & \multirow[b]{2}{*}{$P$ value } \\
\hline & & $\overline{\operatorname{AAD}(n=10)}$ & CAD $(n=15)$ & $\mathbf{A A}(\mathbf{n}=7)$ & PAU $(n=3)$ & \\
\hline Intended completion & $5(13)$ & $2(20)$ & $1(7)$ & $1(14)$ & $1(33)$ & .60 \\
\hline Diameter progression & $17(44)$ & $6(60)$ & $8(53)$ & $2(29)$ & $1(33)$ & .64 \\
\hline Early diameter progression $\dagger$ & $2(5)$ & $2(20)$ & $0(0)$ & $0(0)$ & $0(0)$ & .28 \\
\hline Late diameter progression & $15(38)$ & $4(40)$ & $8(53)$ & $2(29)$ & $1(33)$ & .86 \\
\hline Endoleak & $9(23)$ & $2(20)$ & $4(27)$ & $3(43)$ & $0(0)$ & .58 \\
\hline Type IB & $6(15)$ & $2(20)$ & $2(13)$ & $2(29)$ & $0(0)$ & .91 \\
\hline Type II & $2(5)$ & $0(0)$ & $2(13)$ & $0(0)$ & $0(0)$ & .75 \\
\hline Type III & $1(3)$ & $0(0)$ & $0(0)$ & $1(14)$ & $0(0)$ & .27 \\
\hline dSINE & $4(11)$ & $1(10)$ & $3(20)$ & $0(0)$ & $0(0)$ & .68 \\
\hline Stent-graft kinking & $2(5)$ & $1(10)$ & $1(7)$ & $0(0)$ & $0(0)$ & 1.00 \\
\hline Infection of the FET prosthesis & $1(3)$ & $0(0)$ & $0(0)$ & $1(14)$ & $0(0)$ & .29 \\
\hline Lower body malperfusion & $1(3)$ & $0(0)$ & $0(0)$ & $0(0)$ & $1(33)$ & .09 \\
\hline
\end{tabular}

Values are presented as n (\%). $R+$, Aortic reintervention; $A A D$, acute aortic dissection; $C A D$, chronic aortic dissection; $A A$, aortic aneurysm; $P A U$, penetrating atherosclerotic ulceration; $d S I N E$, distal stent-graft induced new entry; $F E T$, frozen elephant trunk. *Multiple mentions possible. †Early diameter increase was defined as an aortic diameter increase requiring reintervention during the index hospitalization. ‡ॄate diameter increase was defined as an aortic diameter increase requiring reintervention after the index hospitalization. 


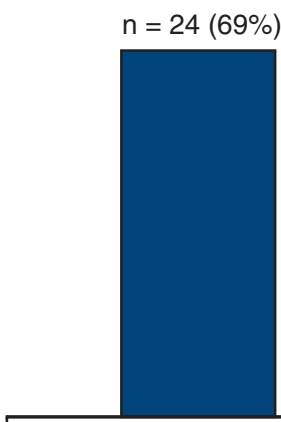

Endovascular

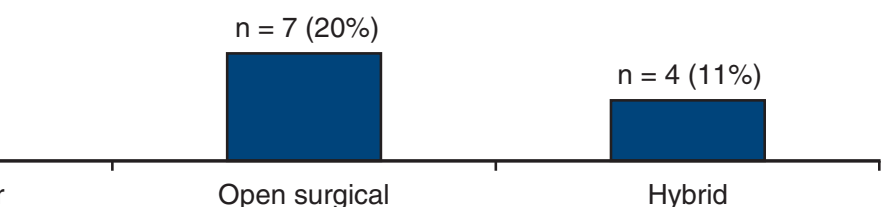

Open surgical

Hybrid

FIGURE 1. Type of aortic reintervention after previous frozen elephant trunk procedure. An endovascular reintervention was performed in 24 patients $(69 \%)$, open surgery was performed in 7 patients $(20 \%)$ and a hybrid approach (endovascular distal extension of the frozen elephant trunk stent-graft shortly followed by open thoracoabdominal replacement) was required in 4 patients $(11 \%)$.

false lumen thrombosis of the thoracic aorta and just marginal perfusion in the false lumen of the abdominal aorta without ectasia of the dissected aortic segments in this patient. The seventh patient developed stent-graft infection and was treated with complete removal of the entire FET prosthesis extensive debridement, and orthotopic vascular reconstruction with self-made tube grafts constructed from a bovine pericardial patch. Lastly, 4 patients $(11 \%)$ underwent a hybrid approach consisting of TEVAR followed by open thoracoabdominal replacement thereafter to ensure stent-graft impermeability and to improve surgical exposure of the proximal anastomosis (Figure 1).

\section{Outcome Characteristics}

Outcome characteristics after the aortic reinterventions are shown in Table 3. No patient developed postoperative stroke, temporary neurologic deficit, or permanent spinal cord injury. One patient developed a temporary sensorimotor deficit of the lower body after TEVAR, but the deficit completely resolved after spinal fluid drainage.

Five patients expired postoperatively. The patient with FET-stent-graft infection died of ongoing sepsis after open surgical reintervention. The second patient underwent open thoracoabdominal aortic replacement. Perioperatively, massive bleeding was observed through multiple spontaneous macropores of the FET stent-graft that were not visible in preoperative CTA. Ultimately, the complete FET stent-graft portion had to be resected to control bleeding, and a vascular prosthesis was anastomosed to the conventional polyester portion of the FET prosthesis. Thoracoabdominal aortic replacement was completed in a common fashion, but the patient died as a result of multiorgan failure in due course. Two other patients also died after open thoracoabdominal aortic replacement in multiorgan failure. The final patient died secondary to thromboembolic lower body malperfusion and visceral ischemia despite the salvage aortic reintervention. In this patient, no thrombus was detected in the FET stent-graft. Although 5 patients died following aortic reintervention, there was no difference in long-term survival between patients with or without aortic reinterventions (Figure 2) (log rank test, $P=.58$ ).

\section{Competing Risk Model}

No risk factors for aortic reinterventions were identified in the competing risk model (Table 4). However, the risk

TABLE 3. Outcome characteristics

\begin{tabular}{|c|c|c|c|c|c|}
\hline \multirow[b]{2}{*}{ Outcome } & \multirow[b]{2}{*}{$\mathbf{R}+(\mathbf{N}=\mathbf{3 5})$} & \multicolumn{3}{|c|}{ Treatment modality } & \multirow[b]{2}{*}{$P$ value } \\
\hline & & Endovascular $(n=24)$ & Open surgery $(n=7)$ & Hybrid* $(n=4)$ & \\
\hline In-hospital mortality & $5(14)$ & $1(4)$ & $4(57)$ & $0(0)$ & .01 \\
\hline Stroke & $0(0)$ & $0(0)$ & $0(0)$ & $0(0)$ & - \\
\hline Temporary neurologic deficit & $0(0)$ & $0(0)$ & $0(0)$ & $0(0)$ & - \\
\hline Permanent spinal cord injury & $0(0)$ & $0(0)$ & $0(0)$ & $0(0)$ & - \\
\hline Temporary spinal cord injury & $1(1)$ & $0(0)$ & $1(0)$ & $0(0)$ & .31 \\
\hline Acute kidney failure & $3(9)$ & $1(4)$ & $2(29)$ & $0(0)$ & .12 \\
\hline Intensive care unit stay (d) & $2(1,3)$ & $2(1,3)$ & $10(1,33)$ & $6(2,29)$ & .08 \\
\hline
\end{tabular}

Values are $\mathrm{n}(\%)$ or median (fist quartile, third quartile). $R+$, Aortic reintervention. *Endovascular distal extension of the frozen elephant trunk stent-graft shortly followed by open thoracoabdominal replacement. 


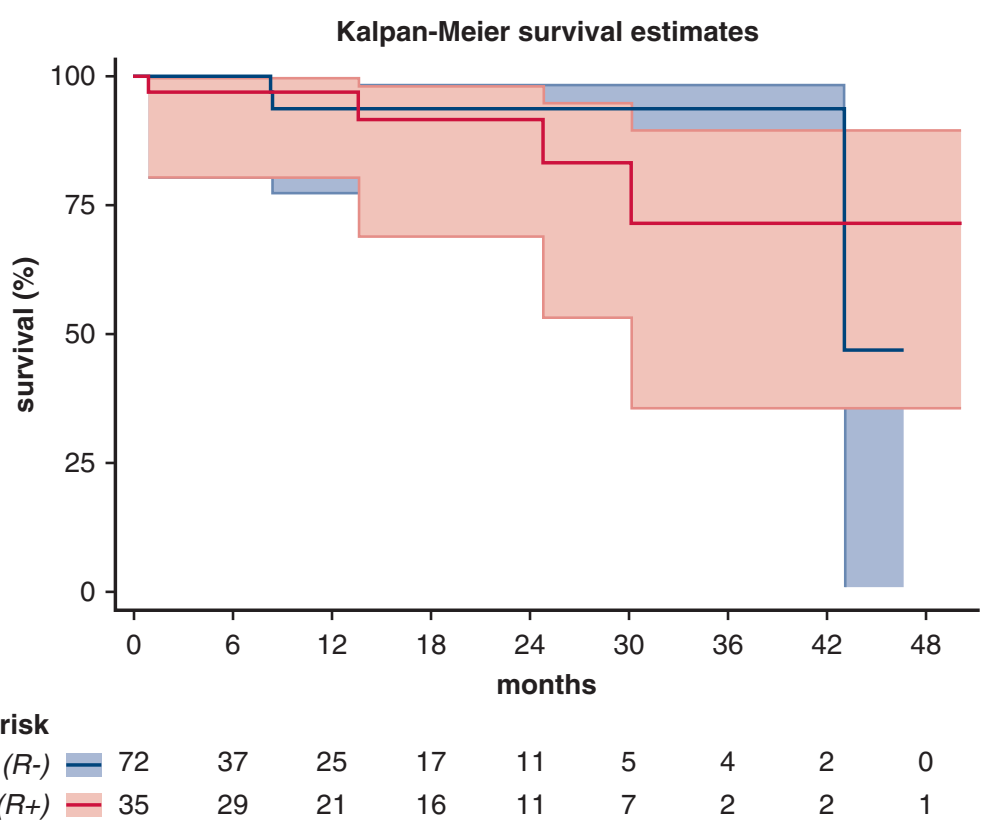

FIGURE 2. Kaplan-Meier analysis of overall survival in patients with aortic reintervention $(R+)$ compared with patients without aortic reintervention $(R-)$ following the frozen elephant trunk (FET) procedure (log rank $P=.58)$. The $95 \%$ confidence intervals are depicted. Five patients expired after aortic reintervention following surgical repair of FET graft infection, lower body malperfusion, spontaneous leakage of the FET stent-graft, and multiorgan failure ( 2 patients), respectively. Three patients expired without aortic reintervention during follow-up for an unknown reason, following a major stroke, and because of pneumogenic sepsis respectively. Note that a survivor bias is present by comparing patients with aortic reinterventions with patients without aortic reinterventions.

for aortic reintervention was $31 \%(95 \%$ confidence interval [CI], 21\%-42\%), 49\% (95\% CI, 35\%-62\%), and 64\% (95\% CI, 44\%-79\%) after 12, 24, and 36 months, respectively (Figure 3).

\section{DISCUSSION}

The most essential findings of this study can be summarized as follows: aortic reinterventions were common and likely after the FET procedure, this study did not identify independent predictors for aortic reintervention and reinterventions were associated with acceptable perioperative morbidity and mortality, and close follow-up of all patients undergoing FET procedure is paramount.

TABLE 4. Competing risk model: Aortic reintervention

\begin{tabular}{lccc}
\hline & \multicolumn{2}{c}{$\boldsymbol{P}$} & \multicolumn{2}{c}{ Subdistribution } & 95\% Confidence \\
value & hazard ratio & interval \\
\hline Age & .32 & 1.02 & $0.98-1.05$ \\
Male & .07 & 2.28 & $0.94-5.48$ \\
Bicuspid aortic valve & .78 & 0.85 & $0.29-2.54$ \\
Marfan syndrome & .26 & 1.68 & $0.69-4.09$ \\
Previous aortic intervention & .59 & 0.76 & $0.29-2.03$ \\
Acute aortic dissection & .91 & 0.92 & $0.21-3.97$ \\
Chronic aortic dissection & .67 & 1.37 & $0.31-6.03$ \\
Aortic aneurysm & .76 & 1.27 & $0.27-6.00$ \\
\hline
\end{tabular}

Chronic health conditions and risk factors were comparable between patients with or without aortic intervention in this study with the exception of a significantly higher rate of chronic renal impairment in patients with no aortic reinterventions. Chronic renal impairment has been reported to significantly reduce long-term survival following thoracic aortic interventions and elective interventions remain high risk for thoracic aortic disease in patients with end-stage renal disease. ${ }^{14}$ In patients with chronic renal impairment, elective thoracic aortic (re-)interventions are not performed liberally at our institution and the individual risks and benefits of any preventative aortic intervention are cautiously analyzed. Nevertheless, a selection bias in this study can be ruled out, because all 13 patients without aortic reintervention but with chronic renal impairment show stable aortic remodeling.

In this series, indications for primary FET implantation varied and the FET procedures were performed in almost half of all patients as a redo aortic intervention, mainly because of a residual aortic dissection following previous Type A aortic dissection repair. This did not influence the incidence of aortic reinterventions following FET implantation. However, in patients with aortic dissection, the rate of false lumen thrombosis was significantly higher in patients without aortic reinterventions. Also, diameter progression was the most common indication for aortic reintervention. This highlights the benefit of distal aortic entry closure in 


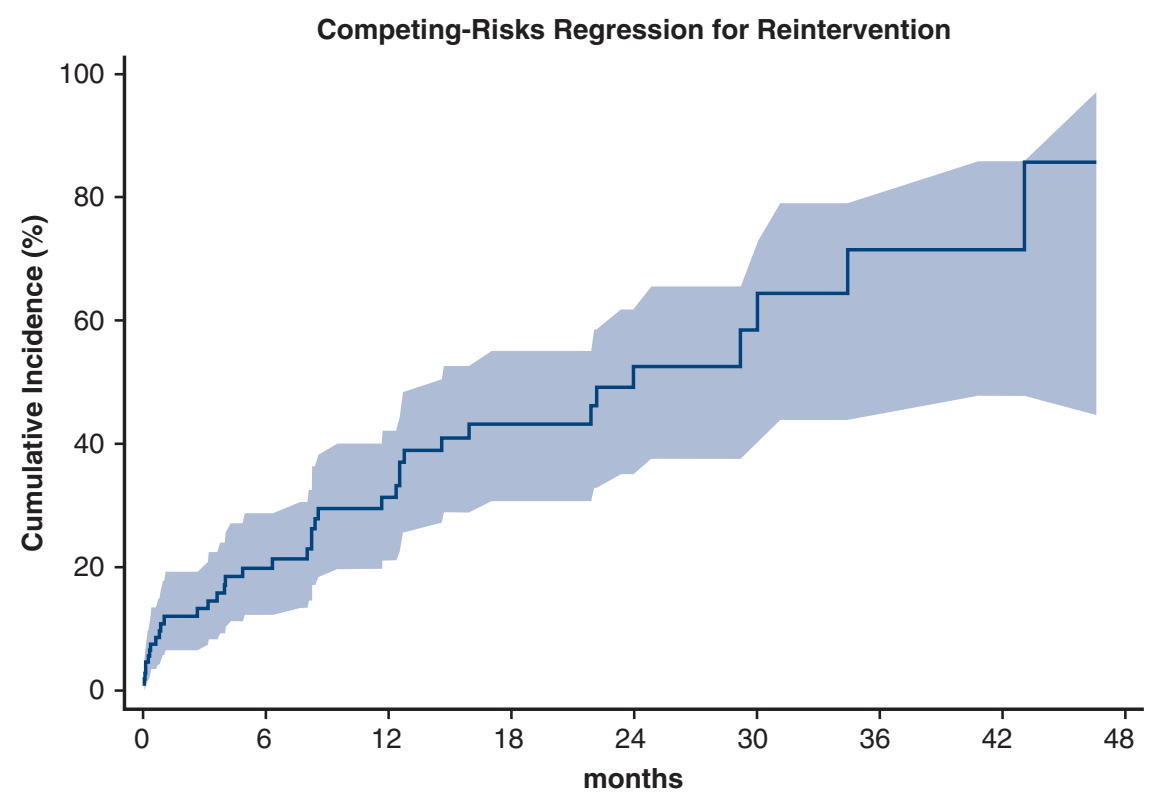

FIGURE 3. Competing risk regression for aortic reintervention following the frozen elephant trunk procedure. The risk for reintervention was $31 \%$ ( $95 \%$ confidence interval [CI], 21\%-42\%), 49\% (95\% CI, 35\%-62\%), and 64\% (95\% CI, 44\%-79\%) after 12, 24, and 36 months, respectively.

patients with aortic dissection to reduce persistent false lumen perfusion and aortic growth. ${ }^{5,6,15}$ In addition, depending on the initial clinical presentation of patients with aortic dissection, a more aggressive arch strategy involving the FET procedure to obtain the most complete primary repair and/or to provide an optimal platform for later second-stage repair, particularly in patients with a large entry tear close to or in the distal aortic arch may be warranted. $2,4,9,16,17$

Endoleak formation following the FET procedure is not uncommon, but was treatable endovascularly to prevent continuous aortic enlargement. ${ }^{7}$ In this collective, $11 \%$ of all patients developed dSINE that also required reintervention to prevent false lumen reperfusion and consequently rapid aortic growth (Figure 4 and Video 1). The complication is not limited to the early postoperative period as reported for conventional TEVAR, but the incidence in this study is relatively higher compared with conventional TEVAR collectives: Dong and colleagues ${ }^{10}$ reported an incidence of 23 out of $650(4 \%)$ TEVAR patients and Jánosi and colleagues ${ }^{18}$ reported an incidence of 9 out of 142 (6\%) TEVAR patients. A mismatch between the fragile dissection membranes and a rigid stent-graft is considered to be the cause for dSINE formation. ${ }^{4,18}$ For this reason, oversizing of the stent-graft portion of the FET prosthesis should be limited. In an acute scenario, Rylski and colleagues ${ }^{19}$ have previously reported 3 possible solutions to calculate the correct stent-graft size that does not exceed the predissection aortic diameter: adding $8 \mathrm{~mm}$ to the true lumen diameter at the first quartile of the dissected aorta, subtracting $3 \mathrm{~mm}$ from the aortic diameter measured between the left carotid and the left subclavian artery, and measuring the maximum diameter of the postdissection true lumen diameter. In a chronically dissected aorta, the dissection membrane is more rigid compared with a mobile membrane in acute dissected aortas and oversizing should be avoided for this reason. ${ }^{18}$ In case of chronic aortic dissections, we always use the smallest possible stent-graft diameter of the FET prosthesis $(24 \mathrm{~mm}, 26 \mathrm{~mm}$, or at most $28 \mathrm{~mm}$ ). Nevertheless, the relatively high rate of dSINE in this study and the lack of less rigid, more flexible stentgraft portions of the FET prosthesis with lower radial force should be taken into account when patients with a chronic dissection membrane require further treatment. Elective distal stent-graft extension with a less rigid, potentially tapered stent-graft might be a preventative option.

At our center, it is standard practice to exclusively use the short version of the FET prosthesis resulting in complete absence of spinal cord injury following the index procedure. ${ }^{2,6}$ In case of extensive descending aortic pathologies that are very likely to require distal extension, we perform distal stent-graft extension shortly after the initial surgery to support spinal cord collateralization thereby preventing spinal cord injury during the aortic reintervention as demonstrated in this study. Also, in patients with extensive downstream aortic pathology, we believe that the FET procedure provides an excellent platform for later endovascular or surgical extension, particularly in patients where affected downstream aortic diameter threshold are not yet reached. Therefore, the so-called risk for reintervention should be used cautiously, because reinterventions may be intended before FET implantation or because reinterventions are anticipated before FET implantation. Nevertheless, the likelihood of aortic reinterventions following the FET 


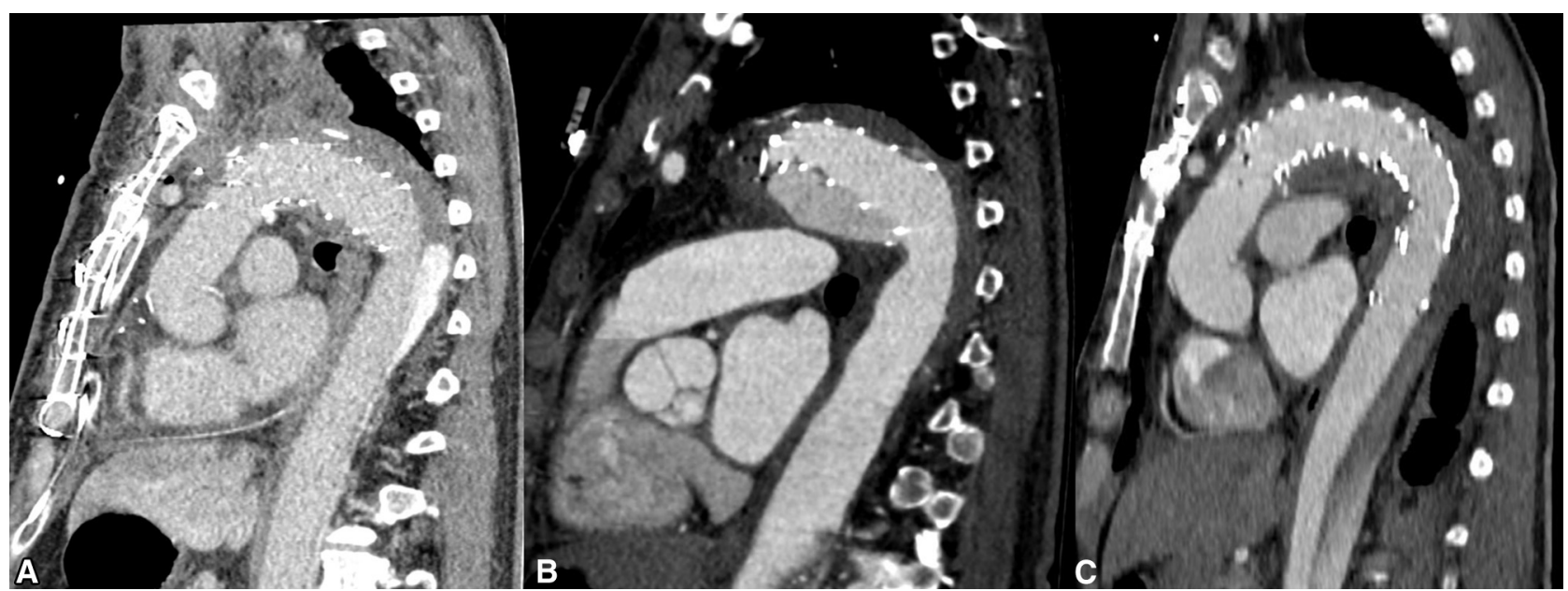

FIGURE 4. A, Exemplary computed tomography angiography of a patient after the frozen elephant procedure for acute aortic dissection. B, The patient developed a distal stent-graft induced entry. C, The patient was successfully treated by endovascular stent-graft extension.

procedure is substantial not only because of predicted aortic changes, but also because of unexpected aortic behavior, stent-graft induced complications particularly dSINE development, or rare cases such as FET graft infection. The multiple causes for reintervention might explain the fact that we were not able to identify any risk factor for aortic reinterventions in our competing risk analysis. This underlines the fact that close and continuous follow-up of all patients undergoing FET procedure independent of the underlying aortic pathology is warranted.

In case aortic reinterventions are necessary, they can be performed endovascularly in most cases. In case open thoracoabdominal aortic replacement is required clamping of the FET stent-graft is possible and a conventional vascular graft can be sewn to the stent-graft portion as demonstrated in this study and as previously shown. ${ }^{20}$ Nevertheless, we have observed spontaneous and unexpected leakage of the FET stent-graft portion during a second-stage thoracoabdominal aortic replacement surgery in this study and in 1 other case (the index surgery was not in our center in this second case). ${ }^{13}$ In addition, Fleissner and colleagues ${ }^{21}$ described 2 incidental late fractures of an FET stent-graft that required reintervention. To prevent perioperative leakage of the FET stent-graft portion and to improve surgical exposure by moving the anastomosis site more distally, a hybrid procedure may be a reasonable approach. Technical progress and new designs by the manufacturers of the FET prosthesis might be necessary in the future to reduce the perioperative risk (eg, spontaneous bleeding) and also the incidence (eg, dSINE formation) of aortic reinterventions.

In this study, 5 patients $(14 \%)$ died following aortic reintervention. One patient developed graft infection, a rare complication following conventional or endovascular aortic treatment with an increasing incidence. ${ }^{11,22}$ Our group recently published our center's integrated standardized therapeutic concept and experience with aortic graft infection. ${ }^{11}$ We were able to show promising short-term results with regard to durability and freedom from reinfection, but a high initial (4 out of 20 patients [20\%] died inhospital) and late mortality (6 out of 20 patients [30\%] died after discharge) secondary to the infectious disease process. Another patient died after perioperative spontaneous and unexpected leakage of the FET stent-graft

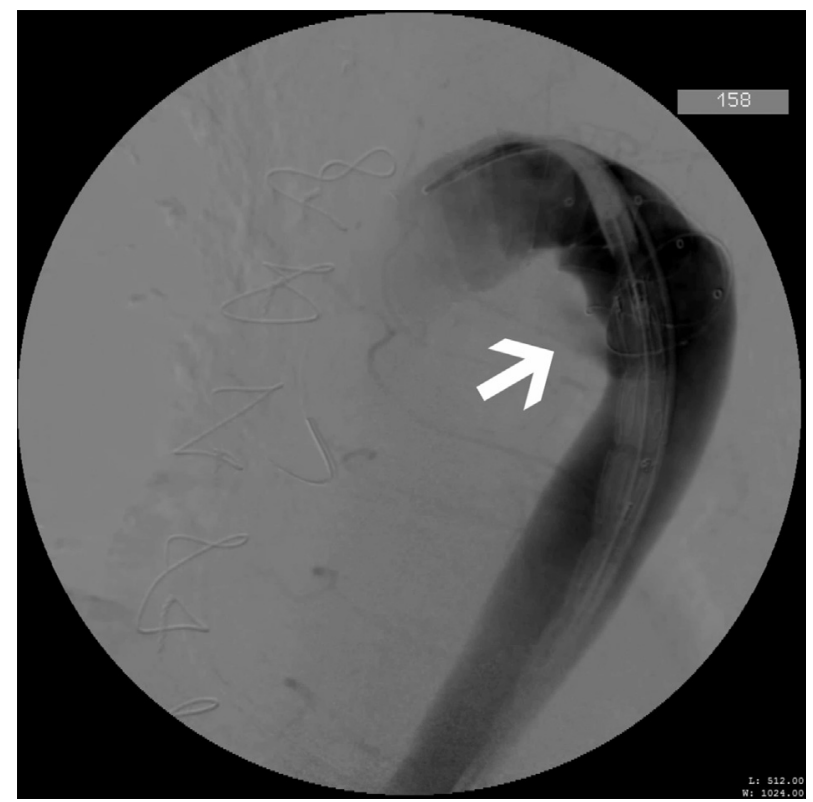

VIDEO 1. Shown is a perioperative angiography of a 70-year-old patient 6 months after frozen elephant treatment for an acute complicated type B aortic dissection. The patient presented for routine follow-up in our aortic clinic and a distal stent-graft-induced new entry (dSINE) was diagnosed. He was scheduled for distal stent-graft extension. The perioperative view shows the dSINE (arrow) and the new stent-graft already in situ. After stent-graft deployment, the dSINE was successfully excluded. Video available at: https://www.jtcvs.org/article/S0022-5223(19)30516-1/fulltext. 
portion. One other patient developed thromboembolic lower body malperfusion following the FET procedure, requiring unexpected endovascular reintervention as a bail-out option that did not save the patient. Taking these 3 unusual postoperative deaths into account and considering that just 2 patients died secondary to conventional open thoracoabdominal aortic aneurysm repair, we believe that aortic reinterventions-open surgical or endovascularcan be performed safely in patients following the FET procedure after adequate patient selection. This is also supported by the fact that late survival was not different in patients with aortic reintervention compared with patients without aortic reintervention.

\section{Limitations and Strengths}

The study is limited by its sample size and its brief follow-up period. However, this study adds valuable knowledge on the high likelihood for aortic reinterventions early after the FET procedure and underlines the need for close aortic follow-up. The literature on aortic reoperations after FET implantation is limited and the patient number of this study is a significant addition to the care of patients with extensive aortic disease. In addition, due to the retrospective character of the study, we were able to identify patients with intended reintervention but we were unable to identify patients with anticipated reinterventions.

\section{CONCLUSIONS}

Aortic reinterventions are common and likely after the FET procedure. The etiology for aortic reinterventions is multifactorial and there are no independent predictors for aortic reinterventions. Technical progress and new designs by the manufacturers of the FET prosthesis might be necessary in the future to reduce the incidence (eg, dSINE formation) and the perioperative risk (eg, spontaneous bleeding) of aortic reinterventions. Nevertheless, when open surgical or endovascular aortic reinterventions are necessary, they are associated with acceptable perioperative morbidity and mortality and the need for reintervention does not affect late survival. Close follow-up of all patients undergoing FET procedure independent of the underlying disease is paramount.

\section{Conflict of Interest Statement}

Dr Czerny and and Dr Rylski received grants/personal fees from TerumoAortic. All other authors have nothing to disclose with regard to commercial support.

\section{References}

1. Czerny M, Rylski B, Kari FA, Kreibich M, Morlock J, Scheumann J, et al. Technical details making aortic arch replacement a safe procedure using the Thoraflex Hybrid prosthesis. Eur J Cardiothorac Surg. 2017;51:115-9.

2. Kreibich M, Berger T, Morlock J, Kondov S, Scheumann J, Kari FA, et al. The frozen elephant trunk technique for the treatment of acute complicated Type B aortic dissection. Eur J Cardiothorac Surg. 2018:53:525-30.
3. Shrestha M, Bachet J, Bavaria J, Carrel TP, De Paulis R, Di Bartolomeo R, et al Current status and recommendations for use of the frozen elephant trunk technique: a position paper by the Vascular Domain of EACTS. Eur J Cardiothorac Surg. 2015;47:759-69.

4. Czerny M, Kreibich M, Morlock J, Kondov S, Scheumann J, Schrofel H, et al. Chronic type B "residual" after type A: what I would do? J Vis Surg. 2018;4:14.

5. Dohle DS, Tsagakis K, Janosi RA, Benedik J, Kühl H, Penkova L, et al. Aortic remodelling in aortic dissection after frozen elephant trunkdagger. Eur J Cardiothorac Surg. 2016;49:111-7.

6. Berger T, Kreibich M, Morlock J, Kondov S, Scheumann J, Kari FA, et al. True-lumen and false-lumen diameter changes in the downstream aorta after frozen elephant trunk implantation. Eur J Cardiothorac Surg. 2018;54 375-81.

7. Luehr M, Peterss S, Zierer A, Pacini D, Etz CD, Shrestha ML, et al. Aortic events and reoperations after elective arch surgery: incidence, surgical strategies and outcomes. Eur J Cardiothorac Surg. 2018;53:519-24.

8. Erbel R, Aboyans V, Boileau C, Bossone E, Bartolomeo RD, Eggebrecht H, et al 2014 ESC Guidelines on the diagnosis and treatment of aortic diseases: document covering acute and chronic aortic diseases of the thoracic and abdominal aorta of the adult. The Task Force for the Diagnosis and Treatment of Aortic Diseases of the European Society of Cardiology (ESC). Eur Heart J. 2014;35: 2873-926.

9. Rylski B, Pérez M, Beyersdorf F, Reser D, Kari FA, Siepe M, et al. Acute non-A non-B aortic dissection: incidence, treatment and outcome. Eur J Cardiothorac Surg. 2017;52:1111-7.

10. Dong Z, Fu W, Wang Y, Wang C, Yan Z, Guo D, et al. Stent graft-induced new entry after endovascular repair for Stanford type B aortic dissection. J Vasc Surg. 2010;52:1450-7.

11. Kreibich M, Siepe M, Morlock J, Beyersdorf F, Kondov S, Scheumann J, et al Surgical treatment of native and prosthetic aortic infection with xenopericardial tube grafts. Ann Thorac Surg. 2018;106:498-504.

12. Maier S, Kari F, Rylski B, Siepe M, Benk C, Beyersdorf F. Selective heart, brain and body perfusion in open aortic arch replacement. J Extra Corpor Technol. 2016:48:122-8.

13. Kreibich M, Siepe M, Beyersdorf F, Czerny M. Spontaneous leakage of the Thoraflex frozen elephant trunk prosthesis. Interact Cardiovasc Thorac Surg. 2019; 28:327-9.

14. Liang NL, Yuo TH, Al-Khoury GE, Hager ES, Makaroun MS, Singh MJ. High mortality rates after both open surgical and endovascular thoracic aortic interventions in patients with end-stage renal disease. J Vasc Surg. 2017:66:991-6.

15. Dohle DS, Jakob H, Schucht R, Janosi RA, Schlosser T, El Gabry M, et al. The impact of entries and exits on false lumen thrombosis and aortic remodelling. Eur J Cardiothorac Surg. 2017;52:508-15.

16. Katayama A, Uchida N, Katayama K, Arakawa M, Sueda T. The frozen elephant trunk technique for acute type A aortic dissection: results from 15 years of experience. Eur J Cardiothorac Surg. 2015;47:355-60.

17. Czerny M. eComment. Re: use of the frozen elephant trunk technique in complicated chronic dissection with porcelain aorta and visceral arteries originating from different lumens. Interact Cardiovasc Thorac Surg. 2016; 22:506.

18. Jánosi RA, Tsagakis K, Bettin M, Kahlert P, Horacek M, Al-Rashid F, et al Thoracic aortic aneurysm expansion due to late distal stent graft-induced new entry. Catheter Cardiovasc Interv. 2015;85:E43-53.

19. Rylski B, Muñoz C, Beyersdorf F, Siepe M, Reser D, Carrel T, et al. How does descending aorta geometry change when it dissects? Eur J Cardiothorac Surg. 2018;53:815-21.

20. Folkmann S, Weiss G, Pisarik H, Czerny M, Grabenwoger M. Thoracoabdomina aortic aneurysm repair after frozen elephant trunk procedure. Eur J Cardiothorac Surg. 2015;47:115-9.

21. Fleissner F, Haverich A, Shrestha M, Martens A. Stent graft perforation of a frozen elephant prosthesis: does design matter? Interact Cardiovasc Thorac Surg. 2015;21:688-90.

22. Revest M, Camou F, Senneville E, Caillon J, Laurent F, Calvet B, et al. Medical treatment of prosthetic vascular graft infections: review of the literature and proposals of a Working Group. Int J Antimicrob Agents. 2015;46:254-65.

Key Words: frozen elephant trunk, aortic surgery, aortic reintervention, TEVAR, distal stent-graft induced new entry 
TABLE E1. Patient characteristics

\begin{tabular}{|c|c|c|c|c|}
\hline Characteristic & All $(N=107)$ & $\mathbf{R}+(\mathbf{n}=\mathbf{3 5})$ & $R-(n=72)$ & $P$ value \\
\hline Age (y) & $66(58,74)$ & $64(54,74)$ & $67(59,73)$ & .14 \\
\hline Male & $69(64)$ & $26(74)$ & $43(60)$ & .14 \\
\hline Hypertension & $96(90)$ & $33(94)$ & $63(88)$ & 1.00 \\
\hline COPD & $13(12)$ & $3(9)$ & $10(14)$ & .54 \\
\hline History of stroke & $10(9)$ & $5(14)$ & $5(7)$ & .29 \\
\hline Coronary artery disease & $32(30)$ & $10(29)$ & $22(31)$ & .83 \\
\hline Chronic renal impairment & $14(13)$ & $1(3)$ & $13(18)$ & .03 \\
\hline Bicuspid aortic valve & $6(6)$ & $4(11)$ & $2(11)$ & .09 \\
\hline Marfan syndrome & $11(10)$ & $4(11)$ & $7(10)$ & 1.00 \\
\hline Previous aortic intervention & $45(42)$ & $15(43)$ & $30(42)$ & 1.00 \\
\hline
\end{tabular}

Values are presented as $\mathrm{n}(\%)$ or median (first quartile, third quartile). $R+$, Aortic reintervention; $R-$, no aortic reintervention; $C O P D$, chronic obstructive pulmonary disease.

TABLE E2. Dissection extension at the time of the frozen elephant trunk procedure

\begin{tabular}{lcccc}
\hline \multicolumn{1}{c}{ Extension } & All $(\mathbf{N}=\mathbf{7 1})$ & $\mathbf{R}+(\mathbf{n}=\mathbf{2 5})$ & $\mathbf{R}-(\mathbf{n}=\mathbf{4 6})$ & \multicolumn{1}{c}{$\boldsymbol{P}$ value } \\
\hline Ascending aorta & $27(38)$ & $7(29)$ & $.34)$ & $35(76)$ \\
Aortic arch & $53(75)$ & $18(75)$ & $25(54)$ & .00 \\
Any supra-aortic vessel & $37(52)$ & $12(50)$ & $42(91)$ & .80 \\
Descending aorta & $63(89)$ & $21(88)$ & $37(80)$ & .68 \\
Abdominal aorta & $55(77)$ & $18(75)$ & $31(67)$ & .60 \\
Any iliac artery & $45(63)$ & $14(58)$ & & .76 \\
\hline
\end{tabular}

Values are presented as $\mathrm{n}(\%) . R+$, aortic reintervention; $R-$, no aortic reintervention. 